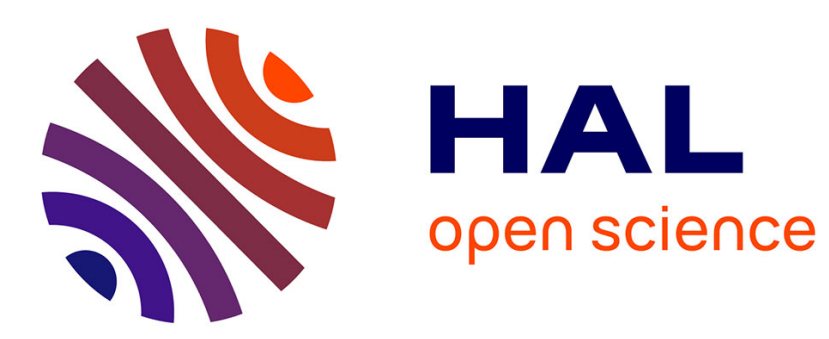

\title{
Perturbation methods in torsion of thin hollow Saint-Venant cylinders
}

Francesco Dell'Isola, Luigi Rosa

\section{To cite this version:}

Francesco Dell'Isola, Luigi Rosa. Perturbation methods in torsion of thin hollow Saint-Venant cylinders. Mechanics Research Communications, 1996, pp.6. hal-00502397

\section{HAL Id: hal-00502397 \\ https://hal.science/hal-00502397}

Submitted on 14 Jul 2010

HAL is a multi-disciplinary open access archive for the deposit and dissemination of scientific research documents, whether they are published or not. The documents may come from teaching and research institutions in France or abroad, or from public or private research centers.
L'archive ouverte pluridisciplinaire HAL, est destinée au dépôt et à la diffusion de documents scientifiques de niveau recherche, publiés ou non, émanant des établissements d'enseignement et de recherche français ou étrangers, des laboratoires publics ou privés. 


\section{PERTURBATION METHODS IN TORSION OF THIN HOLLOW SAINT-VENANT CYLINDERS.}

F. dell'Isola and L. Rosa

Dipartimento di Ingegneria Strutturale e Geotecnica, Università di Roma "La Sapienza", via Eudossiana 18, I-00184 Roma, Italia

(Received 7 April 1995; accepted for print 14 June 1995)

Introduction

In this paper we propose a perturbation method for calculating the fields appearing in Saint-Venant torsion theory in terms of a parameter $(\epsilon)$ characterizing the thickness of the cross section (CS) of Saint-Venant cylinders (SVC). We recover all the classical formulas found by Bredt [1] (see also Vlasov, [2]) as terms of first order in $\epsilon$. Moreover an iterative procedure is obtained which supplies successive corrections to Bredt's formulas useful in the case of CS of "moderate" thickness. The proposed expansion relies on a construction procedure for CS general enough to apply, for instance, to SVC whose doubly connected CS are bounded by ellipses. Thus we can check our perturbative method on the available exact solutions (cf. [3]) of Saint-Venant torsion problem for homothetic elliptic CS. We suggest a procedure to calculate "perturbatively" [5] the fields characterizing the SV torsion problem assuming that the Prandtl function $\phi[6]$ can be expanded in terms of $\epsilon$ :

$$
\phi=\sum_{k=0}^{\infty} \phi_{k} \epsilon^{k}
$$

Let $\mathcal{D}$ be a CS of the SVC, which can be represented as follows: $\mathcal{D}=\mathcal{D}_{1} \backslash \mathcal{D}_{0}$, where $\mathcal{D}_{i}, i=0,1$, are simply connected domains, $\mathcal{D}_{0} \subset \mathcal{D}_{1}$ and $\partial \mathcal{D}_{0} \cap \partial \mathcal{D}_{1}=\emptyset$. Prandtl function $\phi$ is the solution of the following elliptic boundary value problem:

$$
\begin{aligned}
\Delta \phi+2 & =0 \text { in } \mathcal{D} \subset \Pi, \\
\phi & =0 \text { on } \partial \mathcal{D}_{1} \\
\phi & =\bar{\phi} \text { on } \partial \mathcal{D}_{0} \\
\oint_{\partial \mathcal{D}_{0}} \nabla \phi \cdot n & =-2 A_{\partial \mathcal{D}_{0}} .
\end{aligned}
$$

Here $\Pi$ is a plane, $\Delta$ is the Laplace operator, $\nabla$ is the gradient operator, $n$ is the outer normal of the domain $\mathcal{D}_{0}$ and $A_{\theta \mathcal{D}_{0}}$ its area. Because $\phi$ is determined up to a constant we can fix the value of $\phi$ on $\partial \mathcal{D}_{1}$ to be zero. The values of $\phi$ on $\partial \mathcal{D}_{0}, \bar{\phi}$, is an arbitrary constant to be determined using the integral condition (5). Generalizing the results found in [6] we construct a wider class 
of CS. Indeed we consider those sections which are the union of a $\epsilon$-family of curves image of a given curve under a linear (in $\epsilon$ ) homotopy. Once the development for Prandtl function is found we can calculate the development for torsional rigidity $R$, warping $w$ and tangent stress $t$ using the formulas [7],[8],[9].

$$
R=2 G \int_{\mathcal{D}_{1}} \phi+A_{\partial \mathcal{D}_{0}} \bar{\phi}, \quad \nabla w(y)=-\tau(* \nabla \phi(y)+*(y-o)), \quad t=-G \tau * \nabla \phi
$$

where $o \in \Pi, *$ is the $\pi / 2$-rotation operator in $\Pi, y \in \mathcal{D}, G$ is the modulus of elasticity in shear and $\tau$ is the angle of twist.

\section{$\epsilon-$ Families of Cross Sections}

Let $\Gamma_{0}$ be a curve whose parameterization is $r_{0}:[0, l] \rightarrow \Pi$

$$
r_{0}: s \mapsto r_{0}(s) \text {. }
$$

If not misleading we identify $s$ with the arc-length of the curve $\Gamma_{0}$, thus $l$ will be the length of $\Gamma_{0}$. We consider, for Prandtl problem, a family of domains, parameterized by $\epsilon$. The domain $D_{\epsilon}$ is obtained as the union of the curves $\Gamma_{z}, z$-lifted from $\Gamma_{0}$ by the scalar field $\delta$, whose representation is: $\left(r_{0, s}=\frac{d r_{0}}{d s}, \partial \mathcal{D}_{\epsilon}=\Gamma_{0} \cup \Gamma_{1}.\right)$

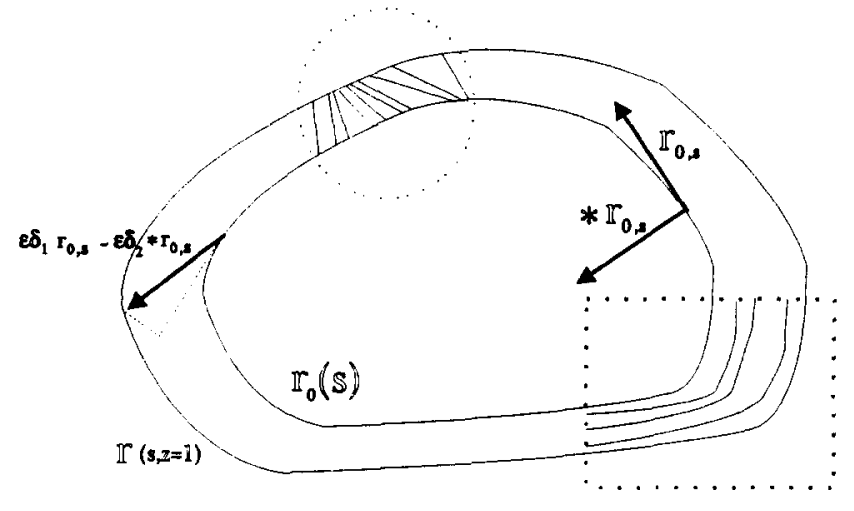

FIG.1

The figure represent a generic $D_{\epsilon}$ domain. The two ortogonal vectors $r_{0, s}$ and $* r_{0, s}$ and the construction (8) are depicted. The circle evidentiates s-constant curves while, in the square, $\mathbf{z}$-constant curves are shown.

Considering the couple $(s, z)$ as a coordinate system on $D_{\epsilon}$ we get the following holonomic basis (when not necessary we omit the explicit s-dependence of the various functions)

$$
\begin{aligned}
& e_{1}(s, z)=\frac{\partial r}{\partial s}=r_{0, s}\left(1+z \epsilon\left(\delta_{1, s}+\delta_{2, s} K\right)\right)+z \epsilon * r_{0, s}\left(K \delta_{1}-\delta_{2, s}\right) \\
& e_{2}(s, z)=\frac{\partial r}{\partial z}=\epsilon\left(r_{0, s} \delta_{1}-* r_{0, s} \delta_{2}\right)
\end{aligned}
$$


$\left(K(s)\right.$ is the curvature of $\left.\Gamma_{0}, \delta_{i, s}=\frac{d \delta_{i}}{d s}, i=1,2\right)$ and the following metric-tensor:

$$
g^{i j}=\frac{1}{g}\left(\begin{array}{cc}
\epsilon^{2}\left(\delta_{1}^{2}+\delta_{2}^{2}\right) & -\left(\epsilon \delta_{1}+z \epsilon^{2}\left(\delta_{1} \delta_{1,0}+\delta_{2} \delta_{2, \Delta}\right)\right) \\
-\left(\epsilon \delta_{1}+z \epsilon^{2}\left(\delta_{1} \delta_{1, \iota}+\delta_{2} \delta_{2,0}\right)\right) & \left(1+z \epsilon\left(\delta_{1,2}+K \delta_{2}\right)\right)^{2}+z^{2} \epsilon^{2}\left(\delta_{2,0}-K \delta_{1,0}\right)^{2}
\end{array}\right)
$$

$g=\epsilon^{2}\left[\epsilon z\left(\delta_{1} \delta_{2, s}-\delta_{1, s} \delta_{2}-K\left(\delta_{1}^{2}+\delta_{2}^{2}\right)\right)-\delta_{2}\right]^{2}$ is the determinant of the metric tensor.

For the sake of completeness we quote here the expression of the gradient and laplacian that will be used in the foregoing [10]:

$$
\nabla \phi=g^{i j} \phi_{, i} e_{j} ; \quad \Delta \phi=g^{i j}\left(\frac{\partial^{2} \phi}{\partial x^{i} \partial x^{j}}-\frac{\partial \phi}{\partial x^{h}}\left\{\begin{array}{c}
h \\
i j
\end{array}\right\}\right)=\frac{1}{\sqrt{g}}\left(\frac{\partial}{\partial x_{i}} \sqrt{g} g^{i j} \phi_{, i}\right)
$$

$\left\{i^{h} j\right\}$ are the Christoffel symbols $, i, j, h=1,2 ; x_{1}=s, x_{2}=z, \phi_{, i}=\frac{\partial \phi}{\partial x^{i}}$.

Formal Expansion of the Prandtl Function

Using (1), (11) and (12.2) eq. (2) becomes:

$$
\begin{aligned}
& \sum_{n=0}^{\infty}\left\{\epsilon^{n} A \phi_{n, z z}+\right. \\
& \epsilon^{n+1} \quad\left(B_{1} \phi_{n, z}+B_{2} \phi_{n, z z}+B_{3} \phi_{n, z z}\right)+ \\
& \epsilon^{n+2} \quad\left[C_{1} \phi_{n, 2}+C_{2} \phi_{n, 2 z}+C_{3} \phi_{n, s}+C_{4} \phi_{n, s z}+C_{5} \phi_{n, s s}\right]+ \\
& \left.\epsilon^{n+3}\left[D_{1} \phi_{n, z}+D_{2} \phi_{n, z z}+D_{3} \phi_{n, 1}+D_{4} \phi_{n, s z}+D_{5} \phi_{n, s s}\right]\right\}= \\
& =-2 \epsilon^{2}\left[\epsilon z\left(\delta_{1} \delta_{2,8}-\delta_{1,8} \delta_{2}-K\left(\delta_{1}^{2}+\delta_{2}^{2}\right)\right)-\delta_{2}\right]^{3} \text {. }
\end{aligned}
$$

Where

- $A=-\delta_{2}$

- $B_{1}=K\left(\delta_{1}^{2}-\delta_{2}^{2}\right)-2 \delta_{1} \delta_{2, s}$

- $B_{2}=z \delta_{1} \delta_{2, s}-3 z \delta_{2} \delta_{1, s}-3 z K \delta_{2}^{2}-z K \delta_{1}^{2}$

- $B_{3}=2 \delta_{1} \delta_{2}$

- $C_{1}=z\left(-2 K^{2}\left(\delta_{1}^{2} \delta_{2}+\delta_{2}^{3}\right)-K\left(\delta_{1}^{2} \delta_{1, s}+3 \delta_{2}^{2} \delta_{1, s}+2 \delta_{1} \delta_{2} \delta_{2, s}\right)-2 \delta_{1} \delta_{1, s} \delta_{2, s}-2 \delta_{2} \delta_{2, s}^{2}-\right.$ $\left.K_{, s}\left(\delta_{1}^{3}+\delta_{1} \delta_{2}^{2}+\delta_{1}^{2} \delta_{2, s s}+\delta_{2}^{2} \delta_{2, s s}\right)\right)$

- $C_{2}=z^{2}\left(-3 K^{2} \delta_{2}\left(\delta_{1}^{2}+\delta_{2}^{2}\right)-\delta_{1, s} K\left(2 \delta_{1}^{2}-6 \delta_{2}^{2}\right)+\delta_{1, s}\left(-3 \delta_{1, s} \delta_{2}+2 \delta_{1} \delta_{2, s}\right)+\delta_{2} \delta_{2, s}\left(4 \delta_{1} K-\delta_{2, s}\right)\right)$

- $C_{3}=\delta_{1}\left(2 \delta_{1} \delta_{2,8}-\delta_{1}^{2} K-\delta_{2}^{2} K-2 \delta_{2} \delta_{1,8}\right)$

- $C_{4}=2 z\left(\delta_{1}^{3} K+\delta_{1} \delta_{2}^{2} K+2 \delta_{1} \delta_{2} \delta_{1, s}-\delta_{1}^{2} \delta_{2, a}+\delta_{2}^{2} \delta_{2, s}\right)$

- $C_{5}=-\delta_{1}^{2} \delta_{2}-\delta_{2}^{3}$

- $D_{1}=-z^{2}\left(\delta_{1}^{2}+\delta_{2}^{2}\right) \delta_{1}^{2} k^{3}-\delta_{2}^{2} K^{3}-3 \delta_{2} \delta_{1,8} K^{2}-2 \delta_{1,8}^{2} K+3 \delta_{1} \delta_{2,0} K^{2}-2 \delta_{2,8}^{2} K-$ $\left(\delta_{1} \delta_{1, s}+\delta_{2} \delta_{2, s}\right) K,+\delta_{1} \delta_{1, s,} K-\delta_{1, s,} \delta_{2,0}+\delta_{2} \delta_{2, s,} K+\delta_{1,8} \delta_{2, s,}$ 
- $D_{2}=z^{3}\left(-\delta_{1}^{4} K^{3}-2 \delta_{1}^{2} \delta_{2}^{2} K^{3}-\delta_{2}^{4} K^{3}-3 \delta_{1}^{2} \delta_{1,3} \delta_{2}, K^{2}-3 \delta_{2}^{3} \delta_{1,8} K^{2}-\delta_{1}^{2} \delta_{1,8}^{2} K-3 \delta_{1,8}^{2} \delta_{2}^{2} K-\delta_{1,8}^{3} \delta_{2}+\right.$ $\left.3 \delta_{1}^{3} \delta_{2, s} K^{2}+3 \delta_{1} \delta_{2}^{2} \delta_{2, s} K^{2}+4 \delta_{1} \delta_{2} \delta_{1,8} \delta_{2, s} K+\delta_{1} \delta_{1,8}^{2} \delta_{2, s}-3 \delta_{1}^{2} \delta_{2, a}^{2} K-\delta_{2}^{2} \delta_{1,8} \delta_{2, s}^{2}+\delta_{2}^{2} \delta_{2, s}^{2} K+\delta_{1} \delta_{2, s}^{3}\right)$

- $D_{3}=z\left(-2 \delta_{1} \delta_{1, s}^{2} \delta_{2}+2 \delta_{1}^{2} \delta_{1, s} \delta_{2, s}-2 \delta_{1,8} \delta_{2, s} \delta_{2}^{2}+2 \delta_{1} \delta_{2} \delta_{2, s}^{2}+\delta_{1}^{4} K_{, s}+2 \delta_{1}^{2} \delta_{2}^{2} K_{, s}+\delta_{2}^{4} K_{, s}+\delta_{1}^{2} \delta_{2} \delta_{1, s s}+\right.$ $\left.\delta_{1, s s} \delta_{2}^{3}-\delta_{1}^{3} \delta_{2, s s}-\delta_{1} \delta_{2}^{2} \delta_{2, s s}\right)$

- $D_{4}=2 z^{2}\left(\delta_{1}^{2} K+\delta_{2}^{2} K+\delta_{1,8} \delta_{2}-\delta_{1} \delta_{2, s}\right)\left(\delta_{1} \delta_{1, s}+\delta_{2} \delta_{2, s}\right)$

- $D_{5}=z\left(\delta_{1}^{2}+\delta_{2}\right)\left(-\delta_{1}^{2} K-\delta_{2}^{2} K+\delta_{1} \delta_{2, s}-\delta_{1, s} \delta_{2}\right)$

Noticing that (12.1) $\left.\nabla \phi \cdot n\right|_{z=0}=-\frac{\delta_{1}}{\delta_{2}} \phi_{, s}+\frac{1}{\delta_{2}} \phi_{, z}$ and using (1), we get for condition (5)

$$
\sum_{n=0}^{\infty} \epsilon^{n} \oint_{\Gamma_{0}}\left\{-\epsilon \frac{\delta_{1}}{\delta_{2}} \phi_{, s}+\frac{1}{\delta_{2}} \phi_{, z}\right\}=-2 \epsilon A_{\Gamma_{0}}
$$

In this way we get for the first three terms of the $\epsilon$-expansion of Prandtl function:

$$
\begin{aligned}
\left(I\left(\Gamma_{0}\right)=\oint_{\Gamma_{0}} \delta_{2}^{-1}, I(s)\right. & \left.=\int_{0}^{s} \delta_{2}^{-1}, \quad J=\frac{2 \delta_{1} \delta_{2, o}+\left(\delta_{2}^{2}-\delta_{1}^{2}\right) K}{\delta_{2}^{2}}\right): \\
\phi_{0}(s, z) & =0, \phi_{1}(s, z)=\frac{2 A_{\Gamma_{0}}}{I\left(\Gamma_{0}\right)}(1-z) \\
\phi_{2}(s, z) & =\frac{(1-z)}{I\left(\Gamma_{0}\right)}\left\{\oint_{\Gamma_{0}} \delta_{2}-\frac{A_{\Gamma_{0}}}{I\left(\Gamma_{0}\right)} \oint_{\Gamma_{0}} J\right\}+\left(z^{2}-z\right) \frac{2 A_{\Gamma_{0}}}{I\left(\Gamma_{0}\right)}\left\{J-2 \delta_{2}^{2}\right\}
\end{aligned}
$$

Torsional Rigidity, Warping and Shear Stress

Using formulas (6) and the expansions $R=\sum_{n=0}^{\infty} R_{n} \epsilon^{n}, w(s, z)=\sum_{n=0}^{\infty} w_{n}(s, z) \epsilon^{n}$ and $t(s, z)=\sum_{n=0}^{\infty} t_{n}(s, z) \epsilon^{n}$, we get:

$$
\begin{aligned}
& R_{0}=0, \quad R_{1}=\frac{4 G A_{\Gamma_{0}}^{2}}{I\left(\Gamma_{0}\right)} \\
& R_{2}=\frac{4 G A_{\Gamma_{0}}}{I^{2}\left(\Gamma_{0}\right)}\left\{I\left(\Gamma_{0}\right) \oint_{\Gamma_{0}} \delta_{2}-\frac{A_{\Gamma_{0}}}{2} \oint J\right\}
\end{aligned}
$$

For the warping

$$
\begin{aligned}
\frac{w_{0}(s, z)}{\tau}= & 2 A_{\Gamma_{0}} \frac{I(s)}{I\left(\Gamma_{0}\right)}+\int_{0}^{s} r_{0} \times r_{0, s} \\
\frac{w_{1}(s, z)}{\tau}= & \frac{A_{\Gamma_{0}}}{I\left(\Gamma_{0}\right)} \int_{0}^{s} J \delta_{2}^{2}+\frac{I(s)}{I\left(\Gamma_{0}\right)}\left\{\oint_{\Gamma_{0}} \delta_{2}-\frac{A_{\Gamma_{0}}}{I\left(\Gamma_{0}\right)} \oint_{\Gamma_{0}} J\right\}-\int_{0}^{s} \delta_{2}+ \\
& z\left\{2 \frac{A_{\Gamma_{0}}}{I\left(\Gamma_{0}\right)} \frac{\delta_{1}}{\delta_{2}}-* r_{0} \cdot r_{0, s}+\delta_{2} r_{0} \cdot r_{0, s}\right\}
\end{aligned}
$$

and finally for the tangential stress 


$$
\begin{aligned}
\frac{t_{0}(s, z)}{G \tau}= & \left(\frac{t_{0 s}}{G \tau}, \frac{t_{0 z}}{G \tau}\right)=\left(2 A_{\Gamma_{0}} \frac{I\left(\Gamma_{0}\right)}{\delta_{2}}, 0\right) \\
\left(\frac{t_{1 s}}{G \tau}, \frac{t_{1 z}}{G \tau}\right)= & \left(2 \frac{A \Gamma_{0}}{I\left(\Gamma_{0}\right)}\left[J / 2+z\left(\delta_{2} \delta_{1,8}-\delta_{1} \delta_{2,0}\right)\right]+\delta_{2}(2 z-1)+\right. \\
& \left.\frac{1}{\delta_{2} I\left(\Gamma_{0}\right)}\left\{\oint_{\Gamma_{0}} \delta_{2}-\frac{A_{\Gamma_{0}}}{I\left(\Gamma_{0}\right)} \oint_{\Gamma_{0}} J\right\}, 2 \delta_{1} A_{\Gamma_{0}} \frac{I\left(\Gamma_{0}\right)}{\delta_{2}}\right)
\end{aligned}
$$

The values $R_{1}, w_{0}$ and $t_{0}$ are the usual ones quoted in the literature [12], [13], [14], they are due to Bredt [1]. We emphasize that for the CS considered in this paper the first non zero contribution to the $z$-component of the shearing stress is of the first order in $\epsilon$.

\section{Conclusions and Perspectives}

Let $\mathcal{D}$ be the section enclosed between two non homothetic ellipses $\Gamma_{0}$ and $\Gamma_{1}$ whose parametric representations are respectively:

$$
\begin{array}{cl}
r_{0}: & {[0,2 \pi] \rightarrow \Pi, \quad r_{0}=(a \cos \varphi, b \sin \varphi)} \\
r: & {[0,2 \pi] \rightarrow \Pi, \quad r=(k a \cos \varphi,(k+q) b \sin \varphi)}
\end{array}
$$

where $k$ is the homothety parameter, $q / k$ is a "homothety defect" which is determined by the angle between the principal axes of $\Gamma_{0}$ and $\Gamma_{1}$ and we choose as $\epsilon$-parameter: $\epsilon:=\frac{k a-a}{a}=\frac{k b-b}{b}=k-1$.

We get for the torsional rigidity

$$
\begin{aligned}
& R_{1}=2 G \pi a^{3} b^{3} \frac{q}{p} \\
& R_{2}=G \pi a^{3} b^{3} \frac{q^{2}}{p^{2}}\left\{\frac{2\left(b^{2}-a^{2}\right)(1-k)}{q}(1+c)+2 a^{2}+c\left[\frac{b^{2}}{2}-\frac{a^{2}(1-k)}{2(k+q-1)}\right]\right\}
\end{aligned}
$$

with $c=\sqrt{\frac{q+k-1}{k-1}}$ and $p=a^{2}-b^{2}+c\left[\frac{\left(b^{2}-a^{2}\right)(k-1)+b^{2} q}{k+q-1}\right]$.

When $q \rightarrow 0$ we find (in agreement with the well known exact formula):

$$
R_{1}=4 \pi G \frac{a^{3} b^{3}(k-1)}{a^{2}+b^{2}}, \quad R_{2}=6 \pi G \frac{a^{3} b^{3}(k-1)^{2}}{a^{2}+b^{2}}
$$

For fixed $a, b$ and $k$ the ratio $\frac{R_{2}}{R_{1}}$ is a function of $q$. When $a=4, b=2$ and $k=1.3$ we get

$$
\frac{R_{2}(k-1)^{2}}{R_{1}(k-1)} \simeq 0.135+0.292 q-0.091 q^{2}+0.122 q^{3}+O\left(q^{4}\right)
$$

so, for example, with $q=0.2$ we find $\frac{R_{2}(k-1)^{2}}{R_{1}(k-1)} \simeq 20 \%$. Therefore the homothety defect increases the value of the second order correction on torsional rigidity.

In the end we want make few comment on the results obtained. Despite the fact that this procedure is general enough to supply all exact solutions available in the literature it is not able to manage the most general CS. The applicability of our expansion procedure and the convergence rapidity of the obtained approximating series depends upon many geometrical factors. We expect that the convergency properties improve if $z$-lifted curves are close to isocurves of the Prandtl function [3]. This condition may not be satisfied by the $\epsilon-$ family of CS we have constructed: indeed eq. 
(8) implies that the s-constant coordinate curves (see Fig.1) are straight lines and in the theory of conformal mapping [15] it is proven that the orthogonal coordinates curves to isocurves of harmonic functions in general are not straight lines. Therefore the proposed expansion method is likely to be valid only when the thickness of the section is "moderate" in the sense that quoted orthogonal coordinate curves can be approximated by straight lines.

At the moment the problem of studying the convergence of the proposed expansion is unsolved. This question could be better understood using the theory of Padé approximants.

\section{Aknowledgment}

We thank prof. A. Di Carlo for having turned our attention to the problem dealt with in this paper.

$\underline{\text { References }}$

1. R. Bredt,Kritische Bemerkungen zur Drehungselastizität, Zeits. Ver. d. Ing. 40,815 (1896)

2. V.Z. Vlasov, tonkostennye uprugyie sterzhni, Fitzmagiz Moskwa (1959) [English translation Thin-walled elastic beams, Israel program for scientific translations, Jerusalem (1961)]

3. S. Timoshenko, J.N. Goodier, Theory of elasticity, Mc Graw-Hill, New York (1951)

4. A. Nayfeh, Perturbation methods, John Wiley and Sons, New York (1973)

5. L. Prandtl, Zur Torsion von prismatischen Stäben, Phys. Zeits. 4, 758 (1903)

6. F. dell'Isola, G. Ruta, Outlook in Saint Venant Theory I: formal expansions for torsion of Bredt-like section, Arch. Mech. Warsaw, 46,6, 1005 (1994)

7. A. Clebsh, Théorie de l'élasticité des corps solides (Traduite par MM. Barré de Saint-Venant et Flamant, avec des Notes étendues de M. Barré de Saint-Venant), Dunod, Paris (1983).

8. I.S. Sokolnikoff, Mathematical Theory of elasticity, McGraw-Hill, New York (1946)

9. A.E.H. Love, A treatise on the mathematical theory of elasticity, Dover, New York (1949)

10. C.E. Weatherburn, An introduction to Riemmanian geometry and the tensor calculus, Cambridge University Press (1963)

11. V. Feodosyev, Soprotivlenie materialov, MIR, Moskwa (1968) [Italian translation: Resistenza dei materiali, Editori Riuniti, Roma (1977)]

12. J.Chase, A.H. Chilver, Strength of materials and structures, Edward Arnold, London (1971)

13. R. Baldacci, Scienza delle costruzioni, UTET, Torino (1970)

14. C. Caratheodory, Theory of functions of a complex variable, Vol. 1,2, Chelsea Publishing Company New York( 1954) 